\title{
Alguns conceitos no ensino da Cosmologia que quase sempre levam a confusão
}

\author{
Some concepts in the teaching of Cosmology that almost always create confusion \\ Jorge Ernesto Horvath ${ }^{*} \sqrt{0}$ \\ ${ }^{1}$ IAG - Universidade de São Paulo, Departamento de Astronomia, São Paulo SP, Brasil.
}

Recebido em 07 de Abril de 2020. Revisado em 09 de Maio de 2020. Aceito em 17 de Maio de 2020.

\begin{abstract}
O ensino da evolução do nosso Universo é um dos temas mais complexos que podem ser abordados na sala de aula. Os conceitos envolvidos estão amiúde muito longe de qualquer intuição, e resultam difíceis de expor e discutir. Apontamos nesta comunicação alguns destes conceitos básicos relativos ao Big Bang, esmiuçando seu contexto científico e os erros mais comuns por parte dos estudantes e seus professores, com a intenção de melhorar o ensino neste ramo das ciências. Os perigos das analogias didáticas utilizadas são apontados em cada caso.

Palavras-chave: Cosmologia; Conceitos no Ensino
\end{abstract}

The teaching of the evolution of the Universe is one of the most complex subjects that can be addressed in the classroom. The involved concepts are frequently very far away of any intuition, and are difficult to expose and discuss. We point out in this communication some of these basic concepts related to the Big Bang, analyzing their scientific content and the most common mistakes made by students and their teachers with the intention of improving the teaching of this branch of Science. The danger of didactical analogies commonly used are pointed out for each case.

Keywords: Cosmology, Teaching Concepts

\section{Introdução}

Dentre todas as ciências exatas, existe um atrativo inegável para os alunos e o público em geral nos temas da Astronomia e Cosmologia. Esta afirmação ouvida insistentemente têm apoio em alguns levantamentos promovidos em vários paises (vide Fróes [1] e referências citadas do Projeto R.O.S.E.) e resulta verossímil. Porém, não é a Astronomia como "ciência natural"a que está sendo almejada, mas antes temas que percorrem a Astrofísica Estelar, Galáxias e a própria história e evolução do Universo. Embora os curricula no Brasil continuem enfatizando a Astronomia "natural", isto é, acessível aos sentidos e plausível de ser abordada empiricamente, o ensino dos conteúdos contemporâneos de Astronomia e Cosmologia resultam muito mais difíceis de abordar, e certamente requerem um grau de abstração e de fundamentação mais ampla, já que praticamente toda a Ciência do século 20 converge neles (vide Kragh [2] para uma discussão geral do papel destes temas na Educação). Temos assim um problema sério nas mãos. De fato não é incomum que os professores contornem temas que são indicados nos PCNs [3], com resultados às vezes frustrantes para os próprios estudantes. E como regra geral, boa parte das abordagens efetivamente ministradas sofre de problemas vários, muitas vezes ancorados na falta de

*Endereço de correspondência: foton@iag.usp.br. conhecimento dos conteúdos e/ou confusões a respeito por parte dos próprios ministrantes.

Abordaremos aqui alguns conteúdos básicos da expansão do Universo, mais precisamente alguns cuja dificuldade conceitual e visualização efetiva criam as maiores confusões na hora da exposição pedagógica. Outros temas muito atuais e relevantes (por exemplo, a expansão acelerada, a Inflação e a constante consmológica) podem ser considerados em uma segunda instância, já que a dificuldade com eles é ainda maior. Como resulta evidente no texto, utilizamos um tratamento o mais direto possível, com a intenção de evidenciar de cheio, sem rodeios, o contexto e os problemas que estamos pretendendo esclarecer.

\section{O Big Bange seus primórdios como ideia física: a descoberta da expansão de Hubble}

A descoberta da chamada expansão de Hubble(nome com o qual ficou conhecida, embora outros autores também colaboraram, e muito, para seu estabelecimento, vide [4] para uma discussão aprofundada) pode ser considerada entre as mais marcantes do século 20 . O desenvolvimento da astronomia óptica e da natureza do átomo tinham então chegado ao ponto onde era possível estudar os objetos astronômicos utilizando os espectrosde absorção 
das "nebulosas"(cuja natureza era ainda incerta !). V. Slipher já tinha constatado que as posições das linhas de absorção nos espectros estavam deslocadas respeito das suas posições medidas em laboratório para muitas destas "nebulosas". Com a melhora nas estimativas das distâncias às "nebulosas"devidas ao trabalho de M. Humason (que empregou métodos mais confiáveis do que o simples tamanho aparente proposta pelo alemão C. Wirtz em 1923), foi possível a determinação de uma relação entre o deslocamento espectral (traduzido este em velocidade pela hipótese de De Sitter de se tratar de efeito Doppler, seção 4.1) e distância. E. Hubble (1929) utilizou aquelas distâncias e também os dados originais de V. Slipher dos desvios das posições das linhas dos espectro, mostrando que para um dado espectro todos os desvios (redshifts) das linhas presentes eram da mesma magnitude, e resultavam proporcionais às distâncias estimadas para as galáxias emissoras, ou seja

$$
v=H_{0} \times D
$$

relação hoje conhecida como Lei de Hubble (Figura1) . Hoje sabemos que a constante de proporcionalidade $H_{0}$ medida originalmente por Hubble era quase 10 vezes maior do que o valor atualmente aceito, já que estava "contaminada" pelos movimentos próprios das galáxias da amostra, as quais eram relativamente próximas (Figura2). Em outras palavras, as velocidades locais estão superpostas ao fluxo global que Hubble desvendou. O deslocamento espectral ficou conhecido como redshift, e as "nebulosas"identificadas como galáxias (no sentido dos Universos-ilha do I. Kant [5]), saga do famoso debate mantido entre F.Curtis e H. Shapley [6].

Embora a amostra original de Hubble consistisse em duas dúzias de objetos, e a maior distância obtida de $\sim 2 M p c$, hoje é possível medir galáxias até distâncias 300 vezes maiores (supernovas e outras "velas"permitem determinar $D$, enquanto grandes telescópios observam

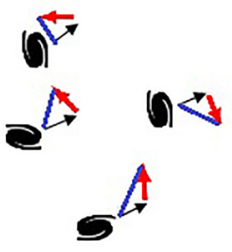

baixo $z$

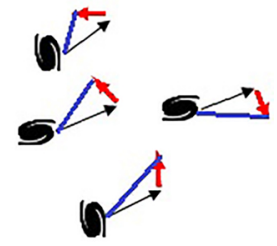

elevado $z$ 1a)

Figura 2: À esquerda (Fig.2a), as galáxias apresentam para distâncias próximas uma componente de movimento próprio (flechas vermelhas) comparável à velocidade de Hubble (flechas pretas). Esta soma está presente muito claramente nos dados originais da expansão, onde as distâncias eram $\leq 2 M p c$. À direita (Fig. 2b), em um conjunto de galáxias mais distantes (elevado $z$ ) o movimento já é dominado pela componente de Hubble, e o movimento próprio resulta quase irrelevante, isto é, a resultante das velocidades é muito mais próxima da componente preta.

os redshifts em espectros muito fracos). O resultado até uns $\sim 500 M p c$ é mostrado na Figura1. Evidentemente, a relação de Hubble se mantém até distâncias muito grandes, e qualquer modelo do Universo deve assim conter razões para que aquela emerja naturalmente deste.

Ficou assim estabelecida uma proporcionalidade entre a velocidade de afastamento das galáxias e a distância. $\mathrm{Na}$ literatura que trata de temas de Cosmologia, costuma-se chamar de fluxo de Hubble a este movimento das galáxias atribuídoà expansão, que decorre de "descontar"os movimentos próprios (locais), tal como mostrado na Figura2. Próximas de nós, para valores baixos do redshift $z$, as galáxias estão "contaminadas"pelo movimento próprio e a componente do fluxo de Hubble é pequena, porque $v=H_{0} \times D$ (note-se que este fato levou a uma deter-

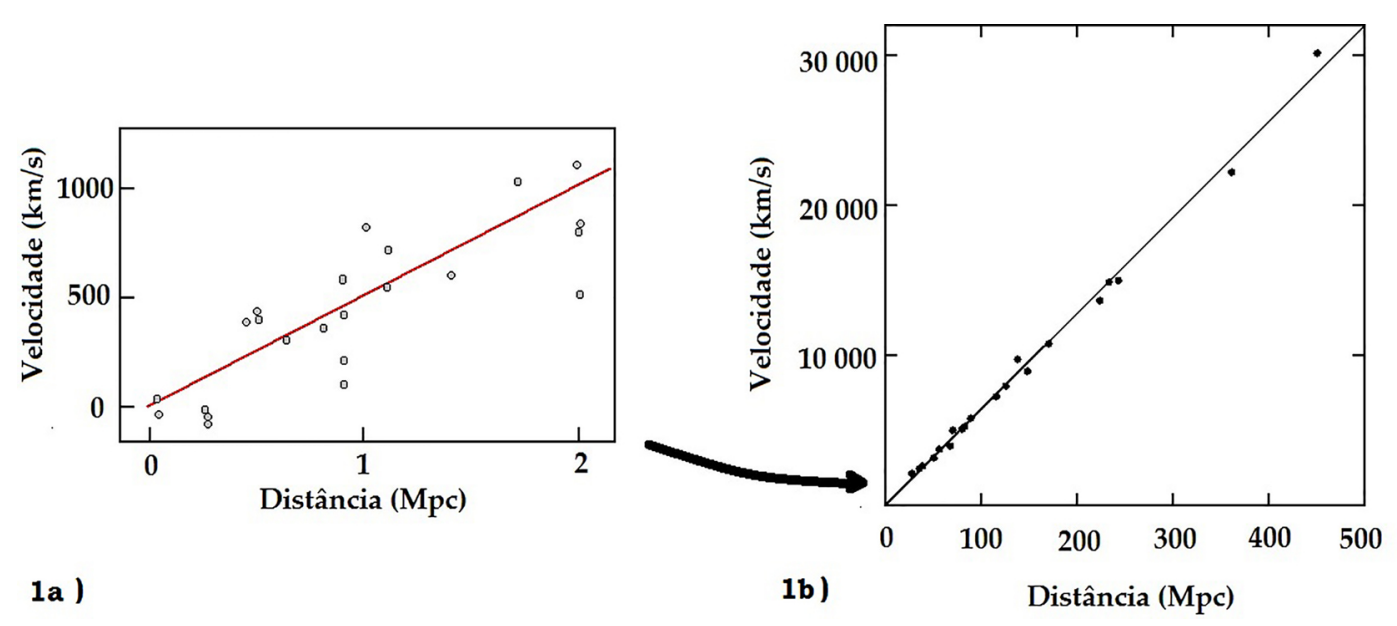

Figura 1: À esquerda (Fig.1a): os dados originais publicados por Hubble em 1929. Note-se a escala de distância e a grande dispersão nas velocidades. À direita (Fig.1b) a relação de Hubble hoje, para amostras muito completas e distâncias e redshifts muito maiores que os medidos no começo do século 20. A amostra original caberia no canto inferior próximo à origem e não está na figura da direita. 
minação erronea de $H_{0}$ ). Quando consideradas galáxias com $z$ mais elevado, a componente de Hubble domina, e o movimento próprio é mínimo respeito deste. A Figura2 ilustra esta diferença importante.

\section{Quantidades próprias e comoveis, a propagação da luz e o redshift}

A Cosmologia nos leva a lidar com objetos (galáxias, quasares, etc.) que trazem para nós uma apreciação diferente dos tempos e distâncias. Consideremos o caso mais simples de observarmos, digamos, uma galáxia cujo espectro mostra um elevado redshift. A velocidade finita da luz $c$, nos diz que estamos vendo esta galáxia não como ela é hoje, mas como era ha um tempo $\Delta t$, da ordem (mas não exatamente!) da distância $D$ dividida por $c$. Mas neste tempo $\Delta t$, tanto a nossa galáxia quanto a observada se moveram no fluxo de Hubble, e assim terá havido uma variação da distância como tempo, que deve ser calculada utilizando a constante de Hubble no passado. Vemos assim que para as observações cosmológicas, há uma mistura indissociável entre coordenadas espaciais e temporais, fato que exige a utilização plena dos conceitos relativísticos em vez dos Newtonianos. Isto deve ser levado em conta quando pensarmos os problemas cosmológicos, mesmo que aparentemente os análogos newtonianos sejam suficientes para a descrição física. Isto sempre é uma fonte de erro.

Um exemplo concreto desta afirmação é a definição da distância a uma galáxia (o artigo clássico de Harrison [7] é de grande utilidade sem excesso de complexidade e resulta muito relevante aqui). Haverá pelo menos duas formas de um observador determinar uma distância, correspondentes a dois sistemas de referência particulares. Uma corresponde a seu sistema de referência "cair" junto com fluxo de Hubble (chamado observador comóvel). Este observador medirá que as distâncias entre ele e as galáxias permanecem fixas ao longo do tempo, denominadas assim distâncias comoveis $\left(d_{c}\right)$. Em contraste, um observador fixo que não acompanha o fluxo de Hubble verá que as distâncias aumentam com o tempo devido à expansão. Estas distâncias são denominadas próprias $\left(d_{p}\right)$. Para ver como estão relacionadas $d_{c}$ e $d_{p}$ consideremos uma galáxia que estava localizada na coordenada $r=r_{1}$ e que emitiu luz em $t=t_{1}$, a qual está chegando até nós hoje (Figura3). A posição da galáxia hoje, arrastada pelo fluxo de Hubble determina sua distância comóvel até nos $d_{c}=a_{0} \times r_{1}$, onde $a_{0}=a\left(t=t_{0}\right)$ é o fator $d e$ escala do Universo hoje, uma quantidade adimensional que caracteriza a expansão (a ser definida e relacionada com os modelos cosmológicos). Se houvéssemos calculado a distância própria no tempo $t_{1}$, esta seria $d_{p}=a_{1} \times r_{1}$, sempre menor do que a atual já que $a_{0}>a_{1}$, porém, esta última não seria uma boa medida, já que inevitavelmente os fótons da luz viajaram enquanto o Universo se expandia (linha diagonal na Figura 3). É por isso que os astrônomos geralmente expressam seus resultados em

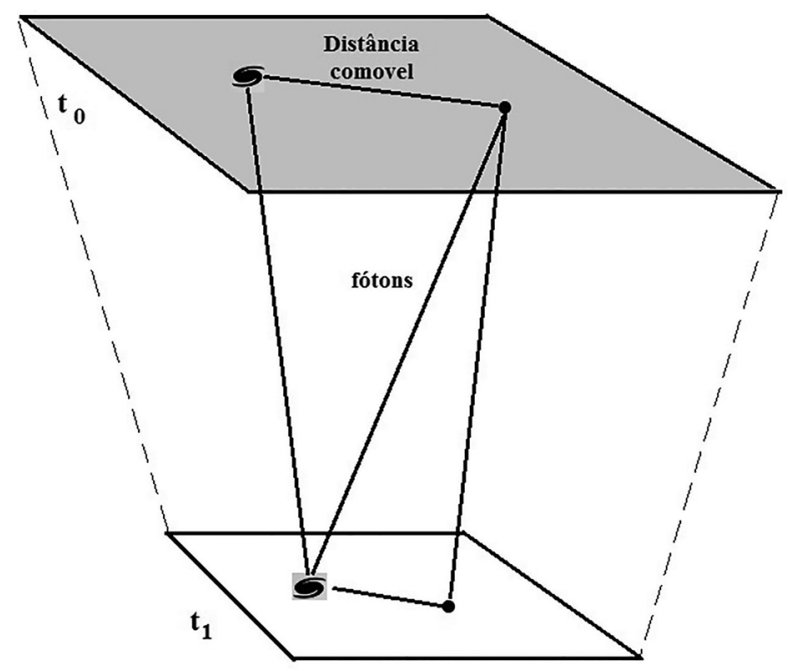

Figura 3: As híper-superfícies no instante $t=t_{1}$ e $t=t_{0}$, mostrando as trajetórias dos raios de luz (diagonal) e as distâncias próprias (calculadas encima da hiper-superfície, representadas por folhas em duas dimensões nesta figura), estas últimas sempre crescente senquanto as comoveis permanecem fixas. Fala-se de "híper"superfícies porque o corte para $\mathrm{t}=$ cte são volumes 3-dimensionais e não verdadeiras superfícies no Universo real.

distâncias comoveis, mesmo que estas não sejam as diretamente observadas. As quantidades próprias e comoveis estão relacionadas entre si pelo fator de escala $a(t)$.

Quando recebemos luz de uma galáxia/quasar distante, podemos escolher a origem do sistema na nossa localização e afirmar que os raios da luz seguem trajetórias radiais. O problema depende somente da coordenadar e o tempo $t$ mas não das coordenadas angulares. Para luz emitida em $t=t_{1}$, a relação entre as nossas coordenadas e as da galáxia emissora é obtida de integrar $d r=c d t / a(t)$, segundo a expressão

$$
r=\int_{t_{1}}^{t_{0}} \frac{c d t}{a(t)}
$$

Este resultado mostra que para conhecer esta relação de coordenadas é imprescindível explicitar um modelo cosmológico, ou seja, uma dinâmica para o Universo que permita conhecer $a(t)$.

Para voltar às observações de Hubble, a atribuição do redshift observado das linhas espectrais à expansão do espaço-tempo (ou "substrato" onde residem as galáxias) permite definir de forma independente do modelo cosmológico aquela quantidade como

$$
(1+z)=\frac{a_{0}}{a_{1}}
$$

quando o fator de escala do Universo seja o atual, $a(t=$ $\left.t_{0}\right) \equiv a_{0}$, não haverá deslocamento algum das linhas, já que então $z=0$. Mas para todo tempo anterior, o redshift cresce conforme a eq. (3), a qual é observável (membro da esquerda), mas que também precisa de um modelo cosmológico onde a dinâmica do $a(t)$ possa ser calculada 
explicitamente e ver se concorda com os dados. Esta é a relação dos observáveis básicos com a expansão e sua modelagem matemática.

Assim, de forma análoga a distância angular $d_{A}$ (definida como a distância entre dois pontos $\delta L$ nos extremos do objeto emissor que têm agora a mesma coordenada radial, que dependem da expansão)o que precisamos saber é sua separação quando os fótons foram emitidos em $t=t_{1}$. Utilizando de novo a equação (3) temos de forma geral que $d_{A} \propto \delta L(1+z)^{-1}$, diretamente mensurável. Mas como $d_{A}$ resulta uma mistura de coordenadas espaciais e redshift não pode, por si própria, constituir um teste definitivo da Cosmologia. Idealmente, estudando um conjunto grande de objetos para os quais tanto o redshiftz quanto a distância angular $d_{A}$ possam ser medidos, poderíamos determinar qual é o modelo de Universo que melhor ajusta às observações. Este tipo de gráfico é conhecido com o nome genérico de diagramas de Hubble, embora com a utilização da $d_{A}$ não é possível ir muito longe até que as incertezas fiquem maiores que as diferenças entre os modelos que pretendem-se pôr em evidência.

Existe assim uma segunda distância importante em Cosmologia, a chamada distância luminosidade $d_{L}$. Esta distância precisa, para a construção de uma escala de distâncias confiável, de "velas-padrão" que permitam utilizar a equação genérica

$$
L \propto \text { Lobservada } \times d_{L}^{2}
$$

que é da mesma forma que aquela utilizada na explicação da definição de lumen, potência luminosa etc., agora generalizada para "velas"("lâmpadas") cosmológicas. A luminosidade observada ésempre na forma de um fluxo, ou quantidade de energia por unidade de área e tempo, que chamaremos de $F$. Sabendo quanto o objeto emite realmente (a luminosidade absoluta $L$ ), teriamos a distância luminosidade extraíndo a raíz do quociente. Note-se que estamos recebendo hoje essa energia, e assim a esfera atravessada pela mesma tem um raio que é a distância comóvel. Chegamos assim de imediato à

$$
F=\frac{1}{4 \pi a_{0}^{2} r^{2}} \frac{1}{(1+z)^{2}} L
$$

para que esta expressão se reduza à convencional $F=$ $\frac{L}{4 \pi d^{2}}$, podemos definir que

$$
d_{L}=a_{0} r(1+z)
$$

e assim, a relação entre estas duas distâncias é simplesmente $d_{L}=d_{A}(1+z)^{2}$. Estas duas quantidades $d_{L} \mathrm{e}$ $d_{A}$ são as mais úteis e utilizadas em geral. Por exemplo, a distância luminosidade é utilizada para construir diagramas magnitude vs. redshift, um tipo comum de diagramas de Hubble, do tipo gerado com as supernovas Ia que apontaram para a expansão acelerada do Universo [8] (não abordada neste artigo). O problema mais sério é o de conferir que as velas-padrão não sofram uma evolução intrínseca, ou seja efeitos que as façam variar sem corresponder meramente ao esmaecimento geométrico pela distância, mas isto requer um aprofundamento astrofísico além da Cosmologia para cada caso.

\section{Esclarecendo os pontos fundamentaisa respeito da expansão de Hubble}

A continuação descrevemos brevemente uma série de erros que aparecem com insistência e explicamos sua correção. Assim, a abordagem em sala de aula poderá ser aprimorada bastante, e simultaneamente o caráter relativístico (no sentido da gravitação, não da Relatividade Restrita) colocado em evidência. Um texto mais completo a respeito pode ser encontrado em Horvath et. al [9]. Os subtítulos escolhidos são deliberadamente impactantes, com o intuito de atuarem como "tarja"para cada assunto.

\subsection{A expansão do Universo não tem nada a ver com o efeito Doppler}

Existe ainda uma séria confusão decorrente da permanência no imaginário da hipótese inicial de De Sitter, quem identificou o deslocamento espectral observado por Slipher e estudado por Hubble com o efeito Doppler, quando na verdade na expansão de Hubble as galáxias não se deslocam respeito do espaço-tempo, mas é este que "estica" com o tempo e as leva junto; a exemplo de um córrego com folhas (galáxias) flutuando.

No tempo de De Sitter, esta diferença crucial não era clara, mas 1 século depois uma quantidade importante de artigos e livros continua perpetuando essa ideia. Se a expansão de Hubble fosse efeito Doppler, a velocidade de afastamento das galáxias não teria como superar a velocidade da luz. Não é isto o que acontece (vide a seguir). Mas o efeito Doppler, linear na velocidade, pode ser pensado como o primeiro termo de uma série de Taylor da função não-linear que descreve a realmente a expansão, e coincide com esta para baixo $z$. Em outras palavras, para baixos redshifts a descrição com a expressão Doppler sempre estará correta, a primeira ordem. Mas isto não impede que não corresponda fisicamente à expansão, e leve a uma confusão "Newtoniana"nos alunos e professores. Uma analogia destas duas situações se mostra na Figura4.

\subsection{As galáxias podem ter velocidades de recessão maiores quec}

Uma série similar de conceptos equivocados tem a ver com a natureza dos instantes iniciais, já que a expansão de Hubble pode ser extrapolada para trás no tempo, e assim concluir que a escala do Universo era $\approx 0$ no "começo". A expressão Big Bang sugere semanticamente uma explosão no espaço-tempo preexistente, enquanto na verdade deve-se pensar na expansão do espaço-tempo em si próprio. Não há local privilegiado ou "centro" do Big 

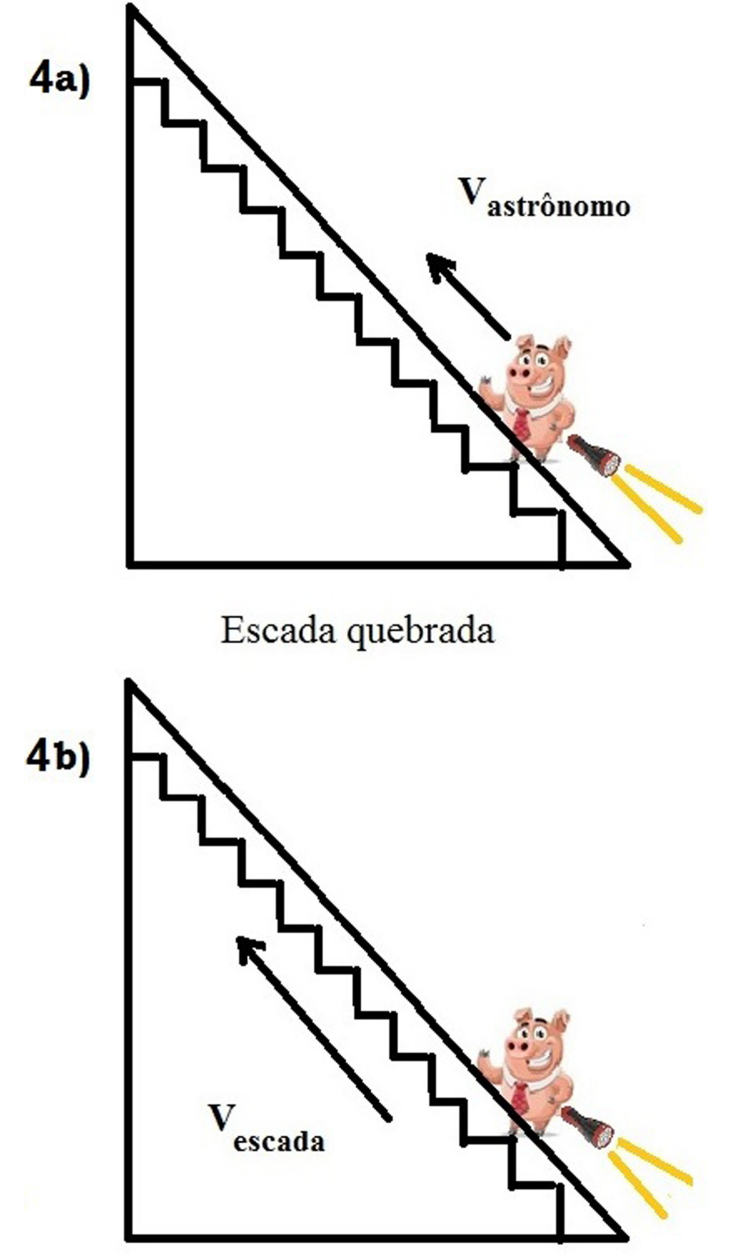

\section{Escada funcionando}

Figura 4: Analogia da expansão de Hubble e o efeito Doppler. Acima (Fig.4a), a interpretação de De Sitter: o substrato (espaçotempo) representado pela escada está fixo (o motor quebrou), e o astrônomo que sobe por ela carregando uma lanterna ilumina o observador enquanto a luz sofre deslocamento Doppler devido à velocidade $\mathrm{V}_{\text {astronomo. Abaixo (Fig.4b) , a escada funciona }}$ (o substrato espaço-temporal se expande), o astrônomo com a lanterna é arrastado e não há "efeito Doppler", antes a lanterna está no referencial próprio e "vê" a expansão de Hubble correspondente à velocidade da expansão $V_{\text {escada }}$.

Bang (como exemplificado com o exemplo do panettone na Figura5, e o pior de todo é que o Universo provavelmente não era "menor" se sua extensão espacial era, de cara, e ainda é infinita. Mas vemos que a nossa região visível ou horizonte era muitíssimo menor. Para dar conta da homogeneidade do Universo, houve, segundo as idéias modernas, um "estiramento"extraordinariamente rápido e muito breve durante achamada era da Inflação, nela o fator de escala $a(t) \propto e^{H t}$. Este modelo leva a uma afirmação que parece surpreendente: não há problema em existirem galáxias cuja velocidade de recessão respeito de nós seja maior que $c$. A restrição da Relatividade Especial ou Restrita se aplica à propagação no espaço-tempo, não

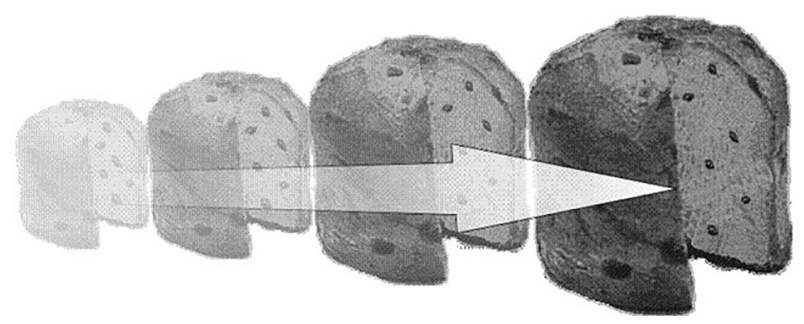

Figura 5: Panettone-modelo do Universo em $3+1$ dimensões que implementa a expansão de Hubble entre "galáxias-passas", viajando por efeito do crescimento da massa no decorrer do tempo. Pode-se medir diretamente a velocidade relativa de afastamento entre passas e conferir que resulta proporcional à distância entre elas (lei de Hubble eq.1). As analogias em $2+1$ dimensões, tais como um balão inflado, são perigosas, já que sugere que $a(t)$ é algo como o raio do balão. O Universo não tem "raio"nesse sentido geométrico. $O$ trabalho de Soares [10] propõe ainda um modelo analógico unidimensional ( $1+1$ dimensões) utilizando uma gominha, o qual é útil para a visualização mas deve ser ponderado convenientemente para evitar confusões.

à expansão do espaço-tempo, que é governado pela Relatividade Geral. A Relatividade Restrita não é aplicável ao Universo e sua dinâmica globalmente, só localmente (acima da híper-superfície). Quando aplicamos a eq.(3) e determinamos um deslocamento, digamos, de $z=3$ para uma galáxia, aparentemente estamos afirmando que essa galáxia se afasta de nos com velocidade $>c$, o qual é verdadeiro, já que o fluxo de Hubble não precisa estar sujeito à Relatividade Restrita. De fato todas as galáxias com $z \geq 1.1$ estão se afastando de nos com velocidades maiores que $c$. A repetência do caráter "absoluto"de $c$ nos cursos de Física provoca este estranhamento. Até cientistas do calibre de R.Feynman chegaram a afirmar que "tinha alguma coisa errada na expansão de Hubble"ao permitir "afastamentos superluminais", possivelmente por não ter refletido o suficiente a respeito da verdadeira natureza deste.

Também leva a várias confusões o fato de não considerar conjuntamente tanto a propagação dos fótons quanto a própria expansão do Universo. Esta é a origem da crença de que não podemos ver uma galáxia que tem velocidade maior que $c$, já qua a luz por ela emitida nunca chegaria até nós. De fato, no caminho da luz, o horizonte cresce e quando os fótons "entram"nele, chegarão até a Terra fatalmente e a galáxia será vista (Figura6). É por isso que vemos galáxias com $z=3,4,5 \ldots$ perfeitamente (vide [11] para uma discussão aprofundada destes assuntos.)

\subsection{O Universo não tem uma "extensão"de 13 bilhões de anos-luz}

Um raciocínio análogo se aplica quando discutida a $e x$ tensão do Universo observável. Para uma dada idade admitida, digamos 13 bilhões de anos, infere-se que o Universo observável tem uns 13 bilhões de anos-luz. Esta não é mais que uma estimativa Newtoniana, no sentido 


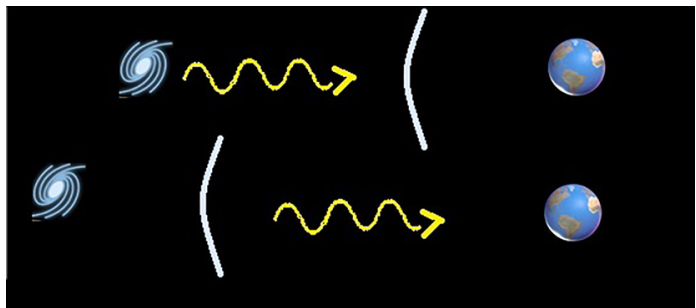

Figura 6: Uma galáxia emite luz, e quando os fótons "entram" no horizonte devem chegar ao observador. Isto acontece porque o Universo visível ("interior"ao horizonte representado pelo curva em branco) cresce com o tempo.A galáxia continua se afastando, mas os fótons que carregam sua imagem entraram no horizonte.

de admitir que a luz se propaga num espaço-tempo (background) fixo, do tipo descrito por Minkowski [5,9]. Porém, se calcularmos a distância até a galáxia quando o fóton chega até nós utilizando um modelo cosmológico em expansão, o resultado e aproximadamente 3 vezes maior. As estimativas que "fixam" o raio de Hubble multiplicando (idade $\times c)$ não levam em conta que enquanto os fótons viajam vemos progressivamente uma fração maior do Universo, e estão erradas numérica e conceitualmente.

\subsection{Os objetos individuais no Universo não "esticam"com a expansão}

Finalmente, existe também o problema de compreender se os objetos dentro do Universo são eles próprios afetados pela expansão. Embora todo o espaço-tempo está se expandindo, as forças que determinam a estabilidade e estrutura da matéria são muito mais do que capazes de equilibrar este efeito. Por exemplo, uma pessoa não se expande com a lei de Hubble porque quando a matéria orgânica que a compõe quiser seguir esta expansão, as forças mecânicas que a mantém estruturada não o permitem. Este mesmo raciocínio vale, por exemplo, para uma estrela ou uma galáxia como um todo, podemos dizer que há uma posição de equilíbrio infinitesimalmente diferente, e assim param de esticar. Os objetos ligados não "esticam" com a lei de Hubble, e mesmo se o fizessem, o resultado seria imperceptível.

\section{Conclusões}

Não é nada fácil o Ensino da Cosmologia, especialmente por introduzir conceitos decorrentes da Teoria da Gravitação (hoje quase sinônimo de Relatividade Geral, mas que poderia se dissociar dela se houver avanços que a superem). Muitos destes são anti-intuitivos, e parecem absurdos a primeira vista, até a pessoas com formação em Ciências que a confrontam pela primeira vez $[1,2,7,12]$. Este trabalho pretende melorar esse descompasso, e trazer aos professores e alunos mais próximos destas ideias. Se levarmos em conta o caráter Newtoniano, se tanto, da maior parte deles, compreende-se como é complexa esta tarefa. Resulta interessante ainda observar que até um conhecimento da Relatividade Restrita pode atrapalhar o Ensino da Cosmologia, pelo menos em uma primeira instância. O remedio existe, mas não é simples nem imediato.

\section{Agradecimentos}

$\mathrm{O}$ autor agradece o apoio financeiro do $\mathrm{CNPq}$ (Brasil) por meio de uma Bolsa de Pesquisa 304932/2018-3. As sugestões de um Relator/a anônimo contribuíram para melhorar a apresentação e contexto do trabalho e são reconhecidas e apreciadas.

\section{Referências}

[1] A. Fróes, Rev. Bras. Ensino Fís. 36, 1 (2014)

[2] H. Kragh, Science \& Education 20, 343 (2011)

[3] Sociedade Brasileira de Física, $P C N+$ Ensino Médio, http://www.sbfisica.org.br/arquivos/PCN_FIS pdf, acessado em 25/05/2020.

[4] A. Bagdonas, J. Zanetic e I. Gurgel, Caderno Bras. Ens. Fís. 35, 97 (2018)

[5] I. Kant, The Universal Natural History and Theories of the Heavens (Aldershot, Ashgate, 2000)

[6] https://www.e-education.psu.edu/astro801/ content/19_p2.html, acessado em 08/05/2020.

[7] E. Harrison, Astrophys. J. 403, 28 (1993)

[8] https://physicsforme.com/2011/10/04/supernovaedark-energy-and-the-accelerating-universe/, acessado em 08/05/2020.

[9] J.E. Horvath, G. Lugones, M. Porto, S. Scarano e R. Teixeira, Cosmologia Física (Livraria da Física, São Paulo, 2006)

[10] D. Soares, Rev. Bras. Ensino Fís. 36, 1 (2014)

[11] A. Bagdonas, Controvérsias envolvendo a natureza da ciência em sequências didáticas sobre cosmologia. Tese de Doutorado, Universidade de São Paulo, SãO Paulo (2015).

[12] C.H. Lineweaver e T.M. Davies, Sci. Am. 292, 36 (2005) 